\title{
Collectivity evolution in the neutron-rich Pd isotopes toward the $N=82$ shell closure
}

\author{
H. Wang,,${ }^{1,2}{ }^{*}$ N. Aoi ${ }^{3}$ S. Takeuchi, ${ }^{2}$ M. Matsushita,,${ }^{2,4, \dagger}$ P. Doornenbal, ${ }^{2}$ T. Motobayashi, ${ }^{2}$ D. Steppenbeck, ${ }^{2, \dagger}$ K. Yoneda, ${ }^{2}$ \\ H. Baba, ${ }^{2}$ L. Cáceres,${ }^{5}$ Zs. Dombrádi, ${ }^{6}$ K. Kobayashi, ${ }^{4}$ Y. Kondo,${ }^{7}$ J. Lee, ${ }^{2}$ K. Li,,${ }^{1,2}$ H. Liu, ${ }^{1,2}$ R. Minakata, ${ }^{7}$ D. Nishimura, ${ }^{8, \ddagger}$ \\ H. Otsu, ${ }^{2}$ S. Sakaguchi, ${ }^{2,}{ }^{\S}$ H. Sakurai, ${ }^{2,9}$ H. Scheit, ${ }^{10}$ D. Sohler, ${ }^{6}$ Y. Sun, ${ }^{1}$ Z. Tian, ${ }^{1}$ R. Tanaka, ${ }^{7}$ Y. Togano, ${ }^{2, \|}$ Zs. Vajta, ${ }^{6}$ \\ Z. Yang, ${ }^{1}$ T. Yamamoto, ${ }^{3}$ Y. Ye, ${ }^{1}$ and R. Yokoyama ${ }^{11}$ \\ ${ }^{1}$ School of Physics and State Key Laboratory of Nuclear Physics and Technology, Peking University, Beijing 100871, China \\ ${ }^{2}$ RIKEN Nishina Center, 2-1 Hirosawa, Wako, Saitama 351-0198, Japan \\ ${ }^{3}$ Research Center for Nuclear Physics, Osaka University, Ibaraki, Osaka 567-0047, Japan \\ ${ }^{4}$ Department of Physics, Rikkyo University, 3-34-1 Nishi-Ikebukuro, Toshima, Tokyo 172-8501, Japan \\ ${ }^{5}$ Grand Accélérateur National d'Ions Lourds, CEA/DSM-CNRS/IN2P3, Caen, France \\ ${ }^{6}$ Institute for Nuclear Research, H-4001 Debrecen, Pf.51, Hungary \\ ${ }^{7}$ Department of Physics, Tokyo Institute of Technology, 2-12-1 Ookayama, Meguro, Tokyo 152-8551, Japan \\ ${ }^{8}$ Department of Physics, Osaka University, Toyonaka, Osaka 560-0043, Japan \\ ${ }^{9}$ Department of Physics, University of Tokyo, 7-3-1 Hongo, Bunkyo, Tokyo 113-0033, Japan \\ ${ }^{10}$ Insitiut für Kernphysik, Technische Universität Darmstadt, 64289 Darmstadt, Germany \\ ${ }^{11}$ Center for Nuclear Study, University of Tokyo, RIKEN campus, Wako, Saitama 351-0198, Japan \\ (Received 11 June 2013; revised manuscript received 20 October 2013; published 19 November 2013)
}

\begin{abstract}
The neutron-rich, even-even ${ }^{122,124,126} \mathrm{Pd}$ isotopes were studied via in-beam $\gamma$-ray spectroscopy at the RIKEN Radioactive Isotope Beam Factory. Excited states at 499(9), 590(11), and 686(17) keV were found in the three isotopes, which we assign to the respective $2_{1}^{+} \rightarrow 0_{g s}^{+}$decays. In addition, candidates for the $4_{1}^{+}$states at 1164(20) and $1300(22) \mathrm{keV}$ were observed in ${ }^{122} \mathrm{Pd}$ and ${ }^{124} \mathrm{Pd}$, respectively. The resulting $E_{x}\left(2_{1}^{+}\right)$systematics are similar to the $\mathrm{Xe}(Z=54)$ isotopic chain and theoretical predictions by the proton-neutron interacting boson model (IBM-2), indicating the magic nature of $N=82$ in the Pd isotopes.
\end{abstract}

DOI: 10.1103/PhysRevC.88.054318

\section{INTRODUCTION}

The existence of a shell structure is one of the most fundamental features of the atomic nucleus. Nuclei possessing magic numbers, corresponding to the complete filling of shells, are relatively stable. Originally, the same set of magic numbers was thought to span over the entire nuclear chart and a variation of protons and neutrons along isotonic and isotopic chains would not effect the magic nature of these nuclei. Studies over the last two decades have, however, revealed that some numbers lose their magicity in certain neutron-rich regions, while new magic numbers could arise. For example, the ${ }^{31,32} \mathrm{Na}$ isotopes were found to have abnormally high masses [1], while for ${ }^{32} \mathrm{Mg}$ a low first $2^{+}$energy [2] together with a large $\gamma$-ray transition probability [3] were found despite its location along the $N=20$ shell closure. Similar magicity losses were found around $N=8$ [4-6] and $N=28$ [7-9].

In the present study, the heavier neutron-rich region around ${ }^{132} \mathrm{Sn}(Z=50, N=82)$ is explored. Recently, the robustness

\footnotetext{
*wanghe@ ribf.riken.jp

${ }^{\dagger}$ Present address: Center for Nuclear Study, University of Tokyo, RIKEN campus, Wako, Saitama 351-0198, Japan.

${ }^{\ddagger}$ Present address: Department of Physics, Tokyo University of Science, 2641 Yamazaki, Noda, Chiba 278-8510, Japan.

${ }^{\S}$ Present address: Department of Physics, Kyushu University, Fukuoka 812-8581, Japan.

"Present address: Department of Physics, Tokyo Institute of Technology, 2-12-1 Ookayama, Meguro, Tokyo 152-8551, Japan.
}

PACS number(s): 23.20.Lv, 27.60.+j, 25.60.-t, 26.30.Hj of the $N=82$ shell closure has attracted much attention due to its relation to the nucleosynthesis in the rapid neutron capture process ( $r$ process) [10]. While ${ }^{132} \mathrm{Sn}$ is known to exhibit typical characteristics of a doubly magic nucleus, such as a high-lying first excited state [11] and a sudden drop in neutron separation energy for the neighboring, heavier isotope [12]; the structure of more proton-deficient nuclei is poorly known. Spectroscopic information in the very neutron-rich region has been extracted only for the $\operatorname{Pd}(Z=46)[13,14]$ and $\mathrm{Cd}(Z=48)$ isotopes [15-18]. However, the persistence of the $N=82$ magicity is questioned within various theoretical approaches $[19,20]$. Shell-model calculations including the tensor interaction suggest that a large $N=82$ shell gap is preserved [19]. In contrast, Hartree-Fock-Bogoliubov (HFB) calculations with the Skyrme force point toward a reduced gap due to a diffused potential caused by the large excess of neutrons [20]. For the $r$ process, some mass models, such as those using HFB with Skyrme force SkP and ETFSI- $Q$ calculations, assuming an $N=82$ shell quenching are in better agreement with the abundance distribution around $A \approx 130$ [21,22].

The most neutron-rich $N=82$ isotone studied experimentally so far is ${ }^{130} \mathrm{Cd}[17,18]$, showing, however, contradictory results. The $Q_{\beta}$ value was better reproduced by mass models assuming a quenched shell gap [17]. However, the first $2^{+}$state $\left(2_{1}^{+}\right)$suggests a good shell closure as the excitation energy of $1.325 \mathrm{MeV}$ [18] is comparable to the even-even $N=82$ isotones above $Z=50$ [23]. Generally, the energy of the first $2^{+}$state $\left[E_{x}\left(2_{1}^{+}\right)\right]$in even-even isotopes provides an indicator for the structural evolution and a qualitative test for a good shell closure [24]. The present study aims at adding more 
information on the nuclear structure in the vicinity of $N=82$, namely to extend the knowledge of $E_{x}\left(2_{1}^{+}\right)$to $N=76,78,80$ for the palladium $(Z=46)$ isotopes.

\section{EXPERIMENT}

The experiment was performed at the RIKEN Radioactive Isotope Beam Factory (RIBF), operated by the RIKEN Nishina Center and the Center for Nuclear Study of the University of Tokyo. To produce secondary cocktail beams via induced in-flight fission, a ${ }^{238} \mathrm{U}$ primary beam impinged on a $0.5-\mathrm{mm}$ thick natural tungsten target located at the entrance of the BigRIPS separator [25,26]. A cocktail beam composed of nuclei around ${ }^{133} \mathrm{Sn}$ was selected and purified by BigRIPS using the magnetic rigidity $(B \rho)$ selection. Two wedge-shaped aluminum degraders with thicknesses of 0.8 and $0.4 \mathrm{~g} / \mathrm{cm}^{2}$, respectively, were located at the F1 and F5 dispersive foci to suppress the transmission of unwanted fission products. Ions passing through BigRIPS were identified event-by-event by measuring their $B \rho$, energy loss $(\Delta E)$ and time of flight (TOF) using standard beam diagnostics detectors [27]. The total intensity of the secondary cocktail beams was $3 \times 10^{4}$ particles per second (pps).

To induce secondary reactions, the cocktail beams were incident on a $1.11-\mathrm{g} / \mathrm{cm}^{2}$ thick ${ }^{9}$ Be secondary target. The average energy of the $\mathrm{Sn}$ isotopes at the center of the secondary target was around $230 \mathrm{MeV} /$ nucleon. Reaction residues were delivered to the ZeroDegree spectrometer [26] for particle identification. Various secondary reaction products were transported through ZeroDegree. Magnetic fields were set to maximize the transmission of ${ }^{125} \mathrm{Pd}$ fragments with full momentum acceptance $(8 \%)$. For the $\mathrm{Pd}$ isotopes, the mass number of the transported ions ranged from $A=119$ to $A=127$. Particle identification was achieved in a similar way as for the secondary beams by measuring $B \rho, \Delta E$, and TOF event-by-event. The atomic number $Z$ and mass-tocharge ratio $A / Q$ were obtained from $\Delta E$-TOF and $B \rho$-TOF correlations, respectively. The $A / Q$ spectrum for $\mathrm{Pd}$ isotopes passing ZeroDegree is displayed in Fig. 1. No gates were applied in the secondary beams. The low contribution of not fully stripped charge states was confirmed by a total kinetic energy $(E)$ measurement using a $\mathrm{LaBr}_{3}(\mathrm{Ce})$ scintillator located at the final focus of ZeroDegree. By using the $E$ information, the fully stripped ions were selected as shown in Fig. 1. The count rate at the final focus was about $3.0 \times 10^{3} \mathrm{pps}$.

Deexcitation $\gamma$ rays emitted from the secondary reactions were measured by the DALI2 spectrometer [28]. It consisted of 186 large-volume $\mathrm{NaI}(\mathrm{Tl})$ scintillation detectors surrounding the reaction target at polar angles from $14^{\circ}$ to $148^{\circ}$ with respect to the beam direction. Gamma-ray energies measured in the laboratory system were corrected for the Doppler-shift based on the individual DALI2 detector angles. The target chamber was covered by a 1-mm-thick $\mathrm{Pb}$ shield to absorb low energy photons originating from atomic processes. The expected energy resolution and efficiencies were determined from GEANT4 simulations [29]. For a $1-\mathrm{MeV} \gamma$ ray emitted at a velocity of $v \approx 0.6 c$, they were $10 \%$ (FWHM) and 20\%, respectively.

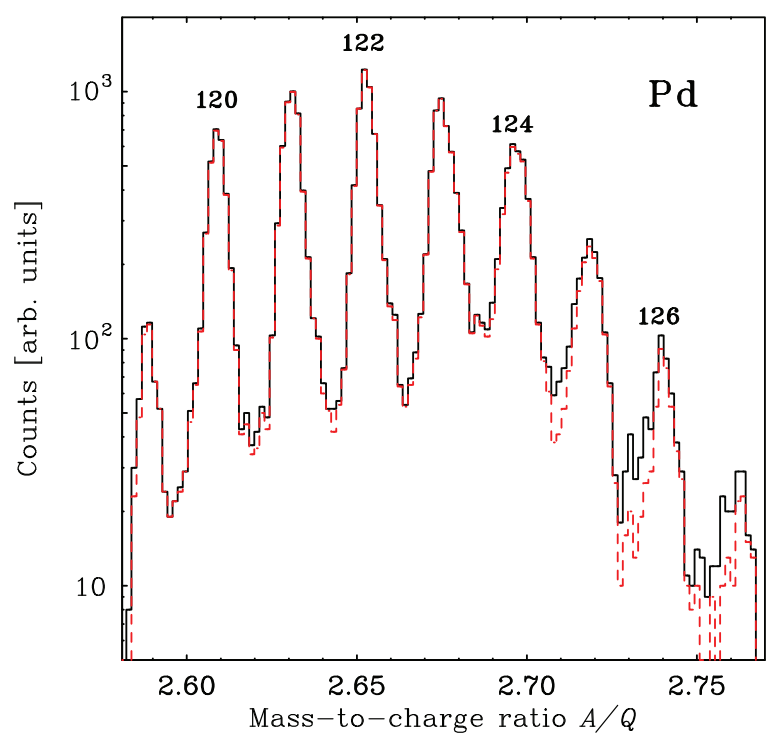

FIG. 1. (Color online) Mass-to-charge ratio $A / Q$ spectrum for $\mathrm{Pd}$ isotopes detected by ZeroDegree. The different even-even isotopes are labeled by their mass numbers. The fully stripped ions are indicated by dashed lines (red). Contributions from not fully stripped charge states are low.

\section{RESULTS}

The Doppler-shift corrected energy spectra of $\gamma$ rays emitted in prompt coincidence with ${ }^{120,122,124,126} \mathrm{Pd}$ isotopes are shown in Fig. 2. To enhance the peak-to-background
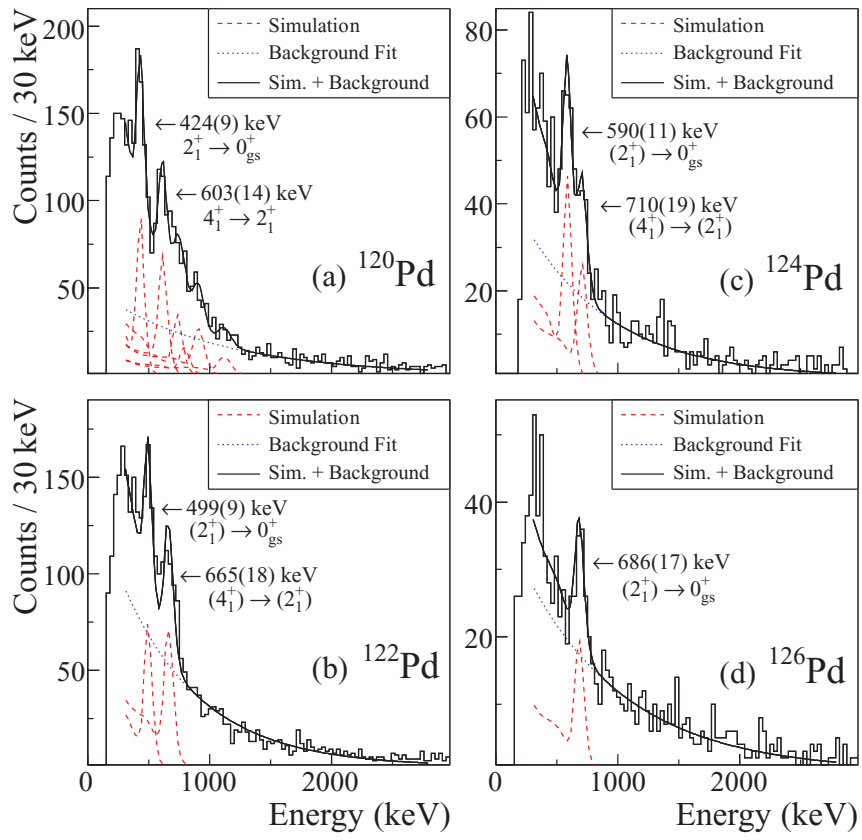

FIG. 2. (Color online) DALI2 $\gamma$-ray energy spectra after correction for the Doppler shift. The spectra in the different panels were obtained by applying coincidence gates with (a) ${ }^{120} \mathrm{Pd}$, (b) ${ }^{122} \mathrm{Pd}$, (c) ${ }^{124} \mathrm{Pd}$, and $(\mathrm{d}){ }^{126} \mathrm{Pd}$ reaction residues detected in the ZeroDegree spectrometer. The spectra are fitted by simulated response functions (red dashed lines) and exponential background (blue dotted lines). The resulting shapes are displayed by the solid lines. 

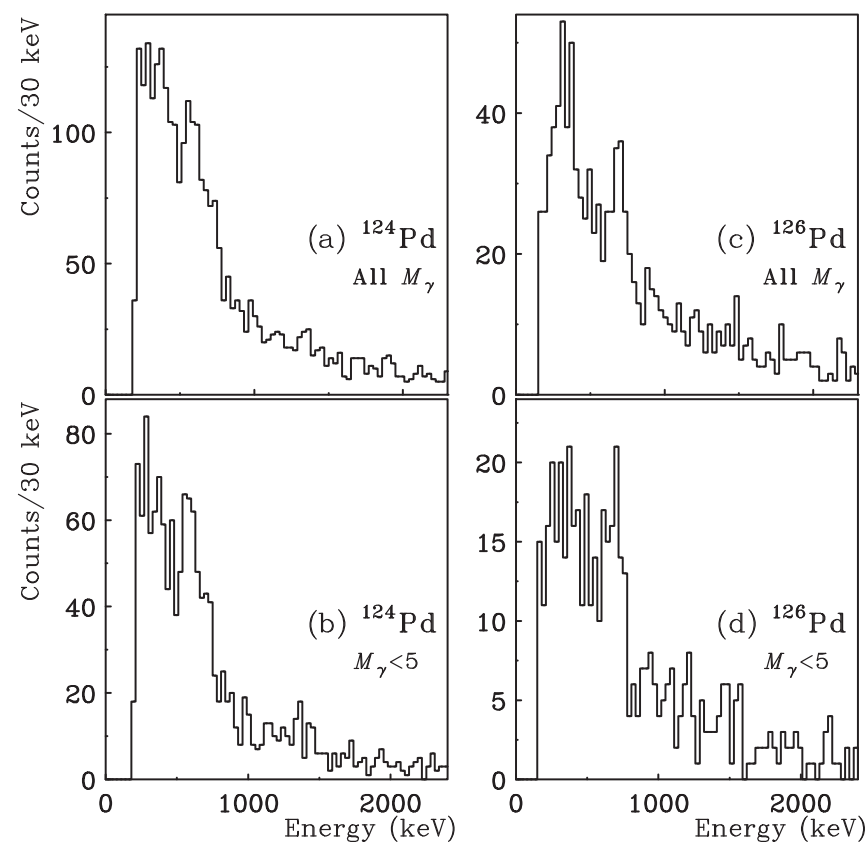

FIG. 3. Gamma-ray energy spectra for $(\mathrm{a}, \mathrm{b}){ }^{124} \mathrm{Pd}$ and $(\mathrm{c}, \mathrm{d}){ }^{126} \mathrm{Pd}$ with different $\gamma$-ray multiplicity conditions. The lower panels show the spectra with the $\gamma$-ray multiplicity cut $\left(M_{\gamma}<5\right)$ and the upper ones for all multiplicities.

ratio, the $\gamma$-ray multiplicity detected in DALI2 was restricted to be lower than 5 for ${ }^{120} \mathrm{Pd}$ to ${ }^{124} \mathrm{Pd}$, but no multiplicity restriction was applied for the ${ }^{126} \mathrm{Pd}$ isotopes due to the lower statistics. The effect of the multiplicity condition is shown in Fig. 3. For ${ }^{120} \mathrm{Pd}$, which served as a test of our setup, two transitions were observed at 424(9) and 603(14) keV, respectively, which correspond to the known $2_{1}^{+} \rightarrow 0_{\mathrm{gs}}^{+}$and $4_{1}^{+} \rightarrow 2_{1}^{+}$decays at 438 and $618 \mathrm{keV}$ [13]. The line-shape of the ${ }^{120} \mathrm{Pd}$ spectrum including the high energy tail for the $4_{1}^{+} \rightarrow$ $2_{1}^{+}$transition was well reproduced by the GEANT4 simulations, taking into account known levels up to spin $J^{\pi}=8^{+}$observed in earlier experiments [14] and the transitions reported in Ref. [13]. The Doppler correction was performed with $\beta$ $(=v / c)$ corresponding to center-of-target energies in both the simulations and analysis. For the $2_{1}^{+}$state in ${ }^{120} \mathrm{Pd}$, a lifetime of around 30 ps can be expected from the systematics in Ref. [30]. This shifts the $2_{1}^{+} \rightarrow 0_{\mathrm{gs}}^{+}$transition from 438 to $431 \mathrm{keV}$ and puts it in agreement with our observed value at $424(9) \mathrm{keV}$. For other excited states, the shift was less pronounced due to the shorter lifetimes. For the $2_{1}^{+} \rightarrow 0_{\mathrm{gs}}^{+}$and $4_{1}^{+} \rightarrow 2_{1}^{+}$transitions, similar intensities were determined by fitting the spectra with the simulated response functions. The stated errors for the peak energies include statistical and systematic uncertainties. This last originates from ambiguities in the energy calibration $(2.5 \mathrm{keV}), \gamma$-ray emission position, as well as the velocity due to the unknown half-lives of the excited states.

For ${ }^{122} \mathrm{Pd}$, two new $\gamma$-ray transitions were observed at 499(9) and at 665(18) keV, respectively. As in the case of ${ }^{120} \mathrm{Pd}$, the two transitions have comparable intensities. The lower energy transition is close in excitation energy to the known $2_{1}^{+}$state in ${ }^{120} \mathrm{Pd}$. Therefore, we assign the $499 \mathrm{keV}$ peak to the $2_{1}^{+} \rightarrow 0_{\mathrm{gs}}^{+}$transition. Applying the same argument for the second peak at $665(18) \mathrm{keV}$, we assign the transition to the $4_{1}^{+} \rightarrow 2_{1}^{+}$decay. Thus, a $E_{x}\left(4_{1}^{+}\right) / E_{x}\left(2_{1}^{+}\right)$ratio $\left(R_{4 / 2}\right)$ of $2.33(8)$ was deduced for ${ }^{122} \mathrm{Pd}$. For the higher energy region, probably several excited states were produced. However, these transitions could not be resolved due to the constraints of our experimental setup.

In the $\gamma$-ray spectra of ${ }^{124} \mathrm{Pd}$ and ${ }^{126} \mathrm{Pd}$, peaks were observed at 590(11) and 686(17) keV, respectively, which we assign to decays from the $2_{1}^{+}$to the $0_{\mathrm{gs}}^{+}$states. A second transition at 710(19) $\mathrm{keV}$ was revealed in addition by fitting the spectrum of ${ }^{124} \mathrm{Pd}$. This transition is assigned to the $4_{1}^{+} \rightarrow 2_{1}^{+}$decay, resulting in a $R_{4 / 2}$ ratio of $2.20(6)$.

\section{DISCUSSION}

Our results extend the systematics of the $2_{1}^{+}$and $4_{1}^{+}$ excitation energies in the Pd isotopes to $N=80$ and $N=78$, respectively. They are shown in Fig. 4 together with all known $E_{x}\left(2_{1}^{+}\right), E_{x}\left(4_{1}^{+}\right)$, and the resulting $R_{4 / 2}$ ratios for the lighter mass even-even Pd isotopes [23] as a function of the neutron number. While ${ }^{96} \mathrm{Pd}$ features a large $2_{1}^{+}$excitation energy due to the $N=50$ shell closure, isotopes in between the

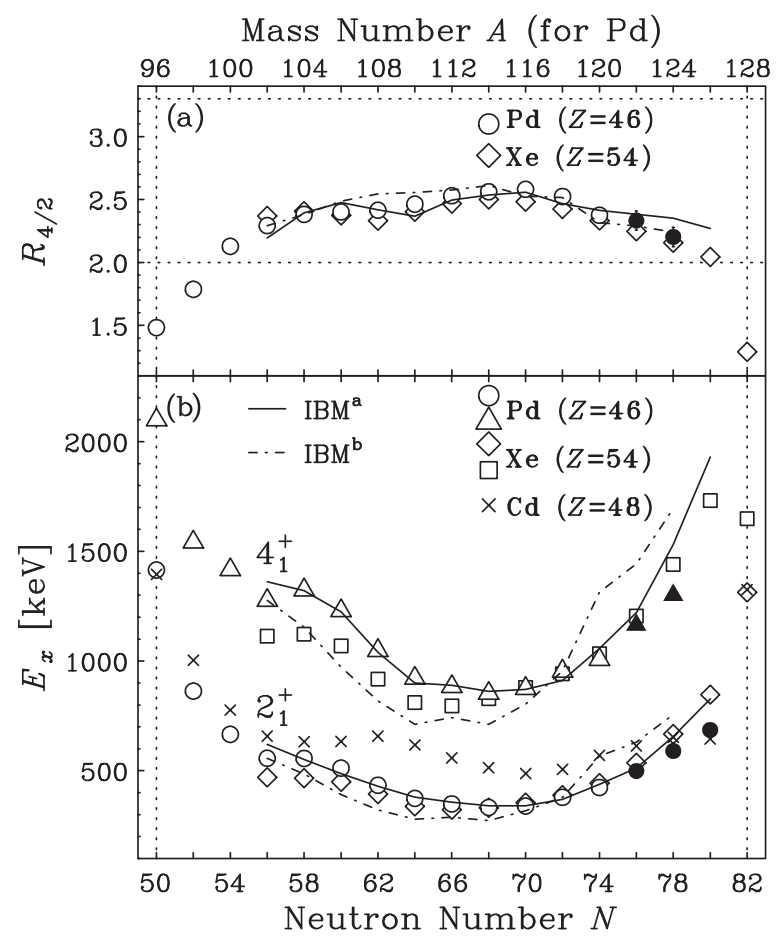

FIG. 4. (a) The $E_{x}\left(4_{1}^{+}\right) / E_{x}\left(2_{1}^{+}\right)$ratios $\left(R_{4 / 2}\right)$ are displayed as a function of the neutron number for Pd (circles) and Xe (diamonds) isotopes. The horizontal dotted lines for values of 2.0 and 3.3 indicate the vibrational and rotational limits, respectively. Panel (b) shows $E_{x}\left(2_{1}^{+}\right)$for $\mathrm{Pd}, \mathrm{Xe}$, and $\mathrm{Cd}$ (crosses) and $E_{x}\left(4_{1}^{+}\right)$for Pd (triangles) and $\mathrm{Xe}$ (squares) isotopes as a function of the neutron number. Open symbols and crosses were taken from Ref. [23] and filled symbols were obtained in this work. Experimental error bars are smaller than the symbol sizes in the lower panel. Solid and dot-dashed lines display two sets of IBM-2 calculations [33,34]. See text for details. 
two major shells $(N=50$ and $N=82$ ) exhibit a parabolic trend with a minimum at $N=68$ in the middle of the shell. Previous experiments already showed a gradual increase of the $2_{1}^{+}$excitation energy commencing at $N=70$. The new data smoothly follows this trend up to $N=80$. We note that the anomalous $E_{x}\left(2_{1}^{+}\right)$pattern of the $\mathrm{Cd}(Z=48)$ isotopes $[15,16]$, shown also in Fig. 4(b) by the crosses, does not appear for the Pd isotopes. In fact, at $N=80$ the $E_{x}\left(2_{1}^{+}\right)$of Pd lies well above the one of ${ }^{128} \mathrm{Cd}$.

The $4_{1}^{+}$excitation energies, as shown in Fig. 4(b), exhibit also a parabolic pattern similar to the one for $E_{x}\left(2_{1}^{+}\right)$, resulting in large $R_{4 / 2}$ ratios in the middle of the shell. At $N=50$, the $R_{4 / 2}$ ratio is well below the vibrational limit (2.0), indicating the closed shell nature of ${ }^{96} \mathrm{Pd}$. The ratio increases as the neutron number and reaches about 2.5 at $N=70$, showing increased deformation. For heavier isotopes, the $R_{4 / 2}$ ratio decreases again. The present results, 2.33(8) and 2.20(6) in ${ }^{122} \mathrm{Pd}$ and ${ }^{124} \mathrm{Pd}$, follow this trend in the $R_{4 / 2}$ systematics, indicating that our data follow the systematical trends pointing to diminishing deformation towards $N=82$.

In Fig. 4, the experimental $E_{x}\left(2_{1}^{+}\right)$and $E_{x}\left(4_{1}^{+}\right)$values for the $Z=54(\mathrm{Xe})$ isotopes are plotted as well for comparison. It has been shown within the $N_{p} N_{n}$ scheme [24] that the four-proton-hole configuration and the four-proton-particle configuration lead to the same nuclear structure signatures. Therefore, provided $N=82$ is a good shell closure, a similar structural evolution as a function of the neutron number is expected for the Pd and $\mathrm{Xe}$ isotopes [31]. Indeed, the $E_{x}\left(2_{1}^{+}\right)$, $E_{x}\left(4_{1}^{+}\right)$, and $R_{4 / 2}$ systematics seen in Fig. 4 exhibit a fair agreement between the $\mathrm{Xe}$ and $\mathrm{Pd}$ isotopes, suggesting a good particle-hole symmetry for protons and hence similar behaviors in the neutron-number dependence for both isotopic chains. Considering the high $2_{1}^{+}$energy at ${ }^{136} \mathrm{Xe}(N=82)$, this similarity suggests a good $N=82$ shell closure in the $\mathrm{Pd}$ isotopes.

The low-lying collective states in medium mass nuclei can be studied with the proton-neutron interacting boson model (IBM-2) [32]. For the Pd isotopes, Kim et al. and Nomura et al. predicted the $E_{x}\left(2_{1}^{+}\right)$and $E_{x}\left(4_{1}^{+}\right)$using this model $[33,34]$. Two different approaches were selected in their calculations. In the first approach, denoted IBM ${ }^{a}$, parameters were determined from microscopic mapping calculations based on the known experimental energies of the even-even $\mathrm{Pd}$ isotopes from ${ }^{102} \mathrm{Pd}$ to ${ }^{114} \mathrm{Pd}$ [33]. In the second approach, denoted $\mathrm{IBM}^{b}$, calculations were performed with a Hamiltonian derived from mapping the potential energy surface of the Skyrme meanfield model $[34,35]$. Both calculations, shown in Fig. 4, can generally reproduce the overall experimental systematic trend.
However, two discrepancies were found between $\mathrm{IBM}^{b}$ and the experimental results. For the lighter $\mathrm{Pd}$ isotopes around $N=60$, the discrepancy is mainly caused by an unexpectedly large deformation using the Skyrme interaction SkM* [34]. A similar discrepancy for this interaction was also observed for the Xe systematics [34]. On the other hand, the discrepancies in both $E_{x}\left(2_{1}^{+}\right)$and $E_{x}\left(4_{1}^{+}\right)$toward $N=82$ may be caused by using a fixed parameter $\chi_{\pi}$ for the entire isotopic chain [34]. A recent attempt using an improved mapping resulted in better overall agreement [36]. As a good $N=82$ shell closure was assumed by choice of model space, the agreement between the IBM-2 calculations and our experimental findings supports again the persistence of a considerable shell gap.

\section{SUMMARY}

In summary, low-lying excited states in ${ }^{122,124,126} \mathrm{Pd}$ have been investigated via in-beam $\gamma$-ray spectroscopy at the RIBF with fast cocktail beams containing mainly ${ }^{132} \mathrm{Sn}$ and ${ }^{133} \mathrm{Sn}$ isotopes. It was found that the $E_{x}\left(2_{1}^{+}\right)$value gradually increases in ${ }^{122,124,126} \mathrm{Pd}$ toward $N=82$, suggesting a good shell closure. For ${ }^{122} \mathrm{Pd}$ and ${ }^{124} \mathrm{Pd}$, the second excited states, which we assigned to $4_{1}^{+}$, were observed, thus confirming the reduction of $R_{4 / 2}$ toward $N=82$. Comparisons with IBM-2 predictions and the excitation energies for $\mathrm{Xe}$ isotopes indicate a magic nature of $N=82$ in the Pd isotopes. However, the slightly lower $E_{x}\left(2_{1}^{+}\right)$values in the Pd isotopes from $N=76$ to $N=80$ compared to the $\mathrm{Xe}$ and the IBM-2 predictions raises the question whether this points to a lower $E_{x}\left(2_{1}^{+}\right)$at $N=82$ and hence a slight weakening of the shell closure. Thus, further studies in this region are desired, in particular on ${ }^{128} \mathrm{Pd}$ itself. This nucleus has recently been identified at the RIBF. Also the behavior of neutron-rich nuclei with lower $Z$ along $N=82$ is of interest, although such studies are an experimental challenge due to their low production cross sections.

Note added. During the review process of this work, we became aware of the recent report by Watanabe et al. [37]. Their results on ${ }^{126} \mathrm{Pd}$ are consistent with our work.

\section{ACKNOWLEDGMENTS}

We express our gratitude to the accelerator staff of RIKEN Nishina Center for providing the intense ${ }^{238} \mathrm{U}$ primary beam and to the BigRIPS team for tuning the secondary beam. We would like to thank Dr. K. Nomura for valuable discussions. The authors Z.D., D.So., and Z.V. were supported by OTKA Grants No. K100835 and No. NN104543.
[1] C. Thibault et al., Phys. Rev. C 12, 644 (1975).

[2] C. Détraz et al., Phys. Rev. C 19, 164 (1979).

[3] T. Motobayashi et al., Phys. Lett. B 346, 9 (1995).

[4] A. Navin et al., Phys. Rev. Lett. 85, 266 (2000).

[5] H. Iwasaki et al., Phys. Lett. B 481, 7 (2000).

[6] H. Iwasaki et al., Phys. Lett. B 491, 8 (2000).

[7] C. M. Campbell et al., Phys. Rev. Lett. 97, 112501 (2006).

[8] B. Bastin et al., Phys. Rev. Lett. 99, 022503 (2007).

[9] S. Takeuchi et al., Phys. Rev. Lett. 109, 182501 (2012).
[10] B. Pfeiffer et al., Nucl. Phys. A 693, 282 (2001).

[11] T. Björnstad et al., Phys. Lett. B 91, 35 (1980).

[12] M. Wang et al., Chin. Phys. C 36, 1603 (2012).

[13] W. B. Walters et al., Phys. Rev. C 70, 034314 (2004).

[14] M. Stoyer et al., Nucl. Phys. A 787, 455 (2007).

[15] T. Kautzsch et al., Eur. Phys. J. A 9, 201 (2000).

[16] L. Cáceres et al., Phys. Rev. C 79, 011301 (2009).

[17] I. Dillmann et al., Phys. Rev. Lett. 91, 162503 (2003).

[18] A. Jungclaus et al., Phys. Rev. Lett. 99, 132501 (2007). 
[19] H. Grawe et al., Eur. Phys. J. A 25, 357 (2005).

[20] J. Dobaczewski, I. Hamamoto, W. Nazarewicz, and J. A. Sheikh, Phys. Rev. Lett. 72, 981 (1994).

[21] B. Chen et al., Phys. Lett. B 355, 37 (1995).

[22] J. Pearson et al., Phys. Lett. B 387, 455 (1996).

[23] NNDC, http://www.nndc.bnl.gov/

[24] R. F. Casten and N. V. Zamfir, Phys. Rev. Lett. 70, 402 (1993).

[25] T. Kubo, Nucl. Instrum. Methods B 204, 97 (2003).

[26] T. Kubo et al., Prog. Theor. Exp. Phys. 2012, 03 C003 (2012).

[27] T. Ohnishi et al., J. Phys. Soc. Jpn. 79, 073201 (2010).

[28] S. Takeuchi et al., RIKEN Accel. Prog. Rep. 36, 148 (2003).
[29] S. Agostinelli et al., Nucl. Instrum. Methods A 506, 250 (2003).

[30] S. Raman, C. Nestor, and P. Tikkanen, At. Data Nucl. Data Tables 78, 1 (2001).

[31] A. Dewald et al., Phys. Rev. C 78, 051302 (2008).

[32] T. Otsuka, A. Arima, and F. Iachello, Nucl. Phys. A 309, 1 (1978).

[33] K.-H. Kim et al., Nucl. Phys. A 604, 163 (1996).

[34] K. Nomura, N. Shimizu, and T. Otsuka, Phys. Rev. C 81, 044307 (2010).

[35] K. Nomura, N. Shimizu, and T. Otsuka, Phys. Rev. Lett. 101, 142501 (2008).

[36] K. Nomura, private communication.

[37] H. Watanabe et al., Phys. Rev. Lett. 111, 152501 (2013). 